\title{
Preparação e caracterização do compósito PVDF/Pani com partículas de níquel
}

\section{Preparation and characterization the composite PVDF/Pani with nickel particles}

\author{
Gabriel da Cruz Dias ${ }^{1,2 *}$, Fernando Rogério de Paula ${ }^{2}$, José Antonio Malmonge ${ }^{2}$ e \\ Luiz Francisco Malmonge ${ }^{2}$ \\ 'Departamento de Ciências - DCI, Universidade Estadual de Maringá - UEM, Campus Regional de \\ Goioerê - CRG, Goioerê, PR, Brasil \\ ${ }^{2}$ Grupo de Polímeros - GPOL, Departamento de Física e Química - DFQ, Faculdade de Engenharia - \\ Câmpus de Ilha Solteira - FEIS, Universidade Estadual Paulista "Júlio de Mesquita Filho" - UNESP, IIha \\ Solteira, SP, Brasil \\ *gcdias2@uem.br
}

\begin{abstract}
Resumo
Neste trabalho partículas de níquel foram incorporadas através da mistura física do pó resultante da mistura PVDF/Pani obtido por síntese química. Filmes homogêneos foram obtidos através da prensagem a temperatura de $180^{\circ} \mathrm{C}$. A partir dos resultados pode-se notar alterações favoráveis e desfavoráveis nas propriedades do compósito após a incorporação e como estas tornam-se dependentes da incorporação. Os resultados de difratometria apresentaram picos característicos tanto da fase $\alpha$ da matriz polimérica e das partículas de níquel, e juntamente com as analises térmicas, que não há alteração na estrutura conformacional e a configuracional. Mesmo apresentando uma boa estabilidade térmica a incorporação indica que além da presença do polímero condutor como o aumento de partículas são prejudiciais às propriedades mecânicas finais da amostra. As micrografias apresentam as partículas de forma dispersas ao longo do filme e determinantes para os valores de condutividade elétrica e obtenção de uma fase magnética.
\end{abstract}

Palavras-chave: polianilina, poli(fluoreto de vinilideno), níquel, compósito, propriedades magnéticas.

\begin{abstract}
In this work nickel particles were incorporated by physically mixing of resulting powder blends of PVDF/Pani, obtained by chemical synthesis. Homogeneous films were obtained by hot pressing at $180{ }^{\circ} \mathrm{C}$. From the results could be noted favorable and unfavorable changes in the properties of composites after incorporation and how this become dependent of the incorporation. The results of diffraction showed the characteristic peaks of both the $\alpha$ phase of the polymer matrix and the nickel particles, and with thermal analysis, no change in the conformational structure and configurational. Even with a good thermal stability incorporating, indicates that besides the presence of the conducting polymer as the increase of particles are harmful to the final mechanical properties of the sample. Micrographs showed particles dispersed throughout the film and determinants to electrical conductivity values and achieving a magnetic phase.
\end{abstract}

Keywords: polyaniline, poly (vinylidene fluoride), nickel, composite, magnetic property.

\section{Introdução}

Tradicionalmente ao estudar se magnetismo e suas propriedades, em particular o ferromagnetismo, está se estudando, por exemplo, elementos inorgânicos como ferro, níquel, cobalto, ligas, cerâmicas e óxidos, os quais apresentam o comportamento magnético, e também estudos quando estes elementos são incorporados em matrizes poliméricas na forma extrínseca ${ }^{[1-3]}$.

Porém a exploração de materiais orgânicos com a descoberta dos polímeros intrinsecamente condutores trouxe uma nova gama de questões no campo da pesquisa como novas propriedades magnéticas e óticas além da possibilidade de materiais mais leves, com alta condutividade e flexibilidade ${ }^{[2-9]}$.

Assim a investigação do comportamento magnético dos polímeros condutores intrínsecos, se inclui o estudo de seus elementos constituintes, como carbono, hidrogênio, nitrogênio, anéis aromáticos e a própria conjunção e conformação da cadeia ${ }^{[10,11]}$ que os caracterizam como materiais leves, e ainda a facilidade por possuírem elétrons desemparelhados, que de alguma forma, podem contribuir para um ordenamento magnética. 
Estes elétrons são oriundos dos processos de oxidação ou redução, devido a inserção de contra-íons, assim como os materiais semicondutores inorgânicos, tais processos são denominados como dopagem e desdopagem.

Nos polímeros condutores intrínsecos, tanto de redução como oxidação, ou seja, a dopagem e desdopagem, irão formar cargas desemparelhadas ao longo da cadeia polimérica, nos quais levam a formação das distorções do retículo e consequentemente da formação de pólarons e bipólarons, respectivamente no interior do gap ${ }^{[12-14]}$ responsáveis pela condução elétrica do material.

Desta forma, os momentos magnéticos dos polímeros condutores intrínsecos são criados, e talvez está aí a dificuldade de se obter a fase ferromagnética. $\mathrm{O}$ mesmo elétron desemparelhado na cadeia, ou seja, o próprio defeito faz com que o polímero apresente um comportamento magnético fraco (pequenas contribuições) como o diamagnetismo, algumas vezes paramagnético ${ }^{[10]}$ e raramente obtenha a fase de ferromagnética.

Entretanto a possibilidade de um polímero condutor intrínseco apresentar um comportamento ferromagnético foi estudada por alguns autores ${ }^{[15-21]}$.

Um aumento na suscetibilidade magnética com o grau de dopagem, seguido de uma diminuição foram observados nos trabalhos de Onoda et al. ${ }^{[16]}$ em pastilhas e filmes de poli(3-fenil-tiofeno) em função da temperatura estes foram atribuídos por transições de pólarons e bipólarons devido ao processo de oxidação.

Amostras de poli(3-dodeciltiofeno) aquecidas acima de $450 \mathrm{~K}$ e posteriormente resfriadas até temperaturas de transição vítrea, evidenciam o comportamento antiferromagnético observado por Sersen et al. ${ }^{[17]}$ através da suscetibilidade magnética devido conversão de pólarons em bipólarons.

O trabalho apresentado por Barta et al. ${ }^{[18]}$ apresentou transições de fase paramagnética para uma fase ordenada, uma fase ferromagnética em amostras de poli (3-alquiltiofeno) induzida pelo aumento do íon dopante $\mathrm{FeCl}_{4}^{-}$.

Medidas de magnetização em função do campo externo aplicado (M-H) foram utilizadas para o estudo da fase ferromagnética de alguns polímeros. Um composto de polianilina e eletroaceptador, tetracianoquinodimetano (Pani/TCNQ), foi estudado por Zaidi et al. ${ }^{[19]}$ este novo polímero possuía uma temperatura crítica de $350 \mathrm{~K}$ e foi observada através de difração de raios X um ordenamento estrutural de seus domínios em função tempo contribuindo no aumento do ferromagnetismo do mesmo.

Long et al. ${ }^{[20]} \mathrm{em}$ seu trabalho foi estudado as propriedades da polianilina, neste foi observado que a magnetização e a suscetibilidade magnética são dependentes do campo aplicado e das concentrações do dopante e transições na suscetibilidade em função da temperatura, transições que confirmam a coexistência das bandas bipolarônicas formadas em diferentes níveis de dopagem.

No trabalho de Pereira et al. ${ }^{[21]}$ o comportamento ferromagnético intrínseco de amostras de poli (3-metiltiofeno) com concentrações insignificantes (ppb) de níquel, cobalto e ferro foi observado não podendo levar a fase magnética desejada, confirmando através de análise de absorção atômica em forno de grafite, que não houve contaminação por meio das partículas.

Dentre os polímeros condutores a Polianilina - Pani, destaca-se devido a sua estabilidade química em condições ambientais no seu estado dopado, a permutação deste estado, com o estado de desdopado, sua fácil síntese química, além de monômero a baixo custo, quando comparado aos demais polímeros condutores ${ }^{[22-26]}$.

Entretanto sua baixa solubilidade na maioria dos solventes orgânicos e sua infusibilidade, tornam-se prejudiciais quanto sua processabilidade, desvantagem das quais dificultam a obtenção de filmes, por exemplo. Além da dificuldade em se obter uma fase metálica, podendo ser alcançada apenas com altos níveis de dopagem para a Pani.

Estes trabalhos em sua maioria envolvem diferentes tipos de dopante e níveis de dopagem do polímero condutor, difícil reprodutibilidade das amostras, além das demais condições de preparo, podendo apresentar diferentes comportamentos magnéticos como diamagnético, paramagnético, ferromagnético e antiferromagnético ${ }^{[15-21]}$.

Assim a produção de misturas e compósitos nas quais associa se estas às propriedades mecânicas e a processabilidade já conhecidas de alguns polímeros termoplásticos, tem acompanhado o estudo dos polímeros condutores nos últimos anos. Nesta, destaca se o poli(fluoreto de vinilideno) - PVDF, por ser facilmente processável em filmes flexíveis e apresentar interessantes propriedades mecânicas, ópticas, térmica e também por ser resistente ao ataque de produtos químicos apresentando uma gama de aplicações ${ }^{[27-29]}$.

Neste trabalho propõe a incorporação de maneira extrínseca de partículas metálicas, na mistura PVDF/Pani ${ }^{[30]}$ para facilitação do comportamento magnético, além de como esta incorporação, pode influenciar nas demais propriedades do material.

\section{Materiais e Métodos}

\subsection{Materiais e síntese}

O PVDF da marca SOLEF 1008/1001 é obtido através da polimerização do fluoreto de vinilideno é obtido na forma de pó e este é utilizado como recebido.

$\mathrm{O}$ monômero de anilina $\left(\mathrm{C}_{6} \mathrm{H}_{5} \mathrm{NH}_{2}\right)$ foi adquirido da SIGMA-ALDRICH e utilizado na síntese após destilação sob vácuo para melhor pureza do material.

Todos os reagentes e solventes (grau para análise) das marcas: SIGMA-ALDRICH, MERCK e SYNTH, as partículas de níquel $(<5 \mu \mathrm{m})$ adquiridas da SIGMA-ALDRICH foram utilizadas conforme recebido.

O compósito foi obtido pela síntese da polianilina em uma solução de PVDF dissolvido em N-N- dimetilformamida $(\mathrm{DMF})^{[30]}$

A Polianilina pode ser obtida pela oxidação do monômero anilina em meio ácido. Primeiramente o PVDF foi dissolvido, sob agitação e aquecimento à $70^{\circ} \mathrm{C}$, em DMF $(10 \mathrm{~m} \% \mathrm{~m} / \mathrm{v})$. Logo após, a solução foi resfriada a temperatura entre $0^{\circ} \mathrm{C}$ e $2^{\circ} \mathrm{C}$ sob agitação, após resfriamento foi acrescentado o monômero anilina e o clorofórmio $\left(\mathrm{CHCl}_{3}\right)$. Em outra solução foi dissolvido o ácido p-tolueno sulfônico (TSA) 
em DMF e acrescentado o agente oxidante persulfato de amônio $\left(\mathrm{NH}_{4}\right)_{2} \mathrm{~S}_{2} \mathrm{O}_{8}$. Esta solução também foi resfriada entre $0^{\circ} \mathrm{C}$ e $2^{\circ} \mathrm{C}$ e depois adicionado clorofórmio.

Após completa homogeneização das duas soluções, a solução contendo o agente oxidante, foi adicionada lentamente a solução contendo o PVDF dissolvido e monômero da anilina.

Em seguida a solução resultante foi deixada à temperatura ambiente por 2 horas, para polimerização do monômero anilina em matriz de PVDF. Logo após, adicionou-se água destilada para que ocorresse a precipitação do produto e cessar a polimerização.

O precipitado foi filtrado e colocado em uma solução de hidróxido de amônio $\left(\mathrm{NH}_{4} \mathrm{OH}\right)$, $1 \mathrm{M}$ por 16 horas sob agitação, a temperatura ambiente para a desdopagem, obteve-se então o produto no estado isolante.

Após este período o produto foi novamente filtrado e levado para secar em vácuo dinâmico por $24 \mathrm{~h}$ e em seguida colocado na estufa a $70^{\circ} \mathrm{C}$ também por $24 \mathrm{~h}$. No processo de redopagem o produto foi colocado sob agitação em solução de ácido clorídrico $(\mathrm{HCl}) 0,05 \mathrm{M}$, por aproximadamente $30 \mathrm{~min}$ e novamente filtrado, e depois pelo processo de secagem e obteve-se então o produto novamente no estado condutor.

As quantidades utilizadas para a obtenção da mistura foram: razão em massa de $\mathrm{PVDF} /$ anilina $=2$, e as razões molares oxidante/anilina $=1$ e TSA/anilina $=2$. A fração de clorofórmio $(\phi)$ [volume de clorofórmio/ (massa de PVDF + massa oxidante) $]=20 \mathrm{ml} / \mathrm{g}$. A Figura 1 apresenta um diagrama esquemático para obtenção das amostras.

\subsection{Obtenção dos filmes e métodos de caracterizações}

Havia duas possibilidades de incorporar as partículas de níquel na mistura. A primeira seria introduzir as partículas durante a síntese no processo de polimerização. A segunda ocorre através da incorporação física, ao adicionar as partículas já com o material sintetizado.

Inicialmente tentou-se incorporar as partículas de níquel durante a obtenção da mistura polimérica, porém na preparação do compósito a utilização de agitadores magnéticos típico de uma síntese não seria aplicável, pois o mesmo não poderia interagir com as partículas, como aprisioná-las no fundo do recipiente. Assim foi utilizado um agitador mecânico.

Foram preparados vários compósitos, com diferentes quantidades de partículas de níquel, tanto para a mistura desdopada como redopada. Entretanto os compósitos obtidos por esse procedimento não apresentaram resultados com o comportamento magnético diferente de um diamgnetismo.

Através Figura 2 nota-se que o compósito quando redopado apresenta um comportamento característicos de um fraco magnetismo, ou não magnetismo, sugerindo que as partículas passasse a interagir com os demais solventes e formando diferentes ions, dos quais não oberva-se comportamento ferromagnético, característico do níquel metálico incorporado ${ }^{[31]}$.

Assim a incorporação ocorre misturando-se o pó resultante da síntese do polímero diretamente com as partículas de níquel, obtendo o compósito PVDF/Pani/Ni.

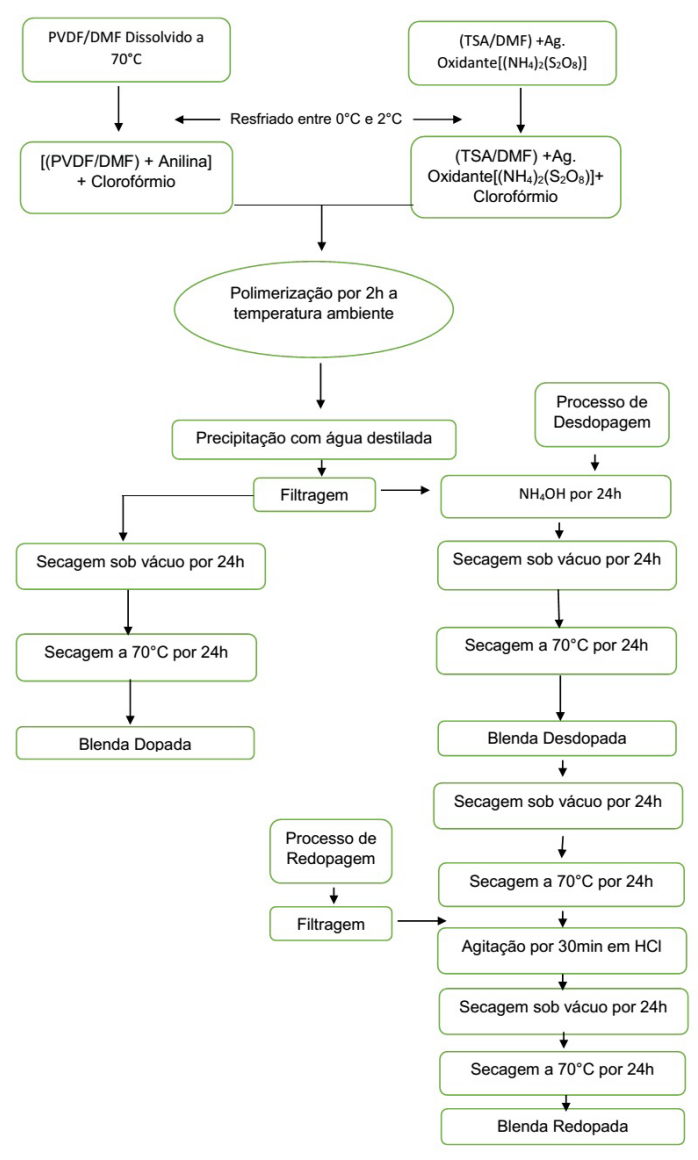

Figura 1. Diagrama esquemático para obtenção da mistura PVDF/Pani.

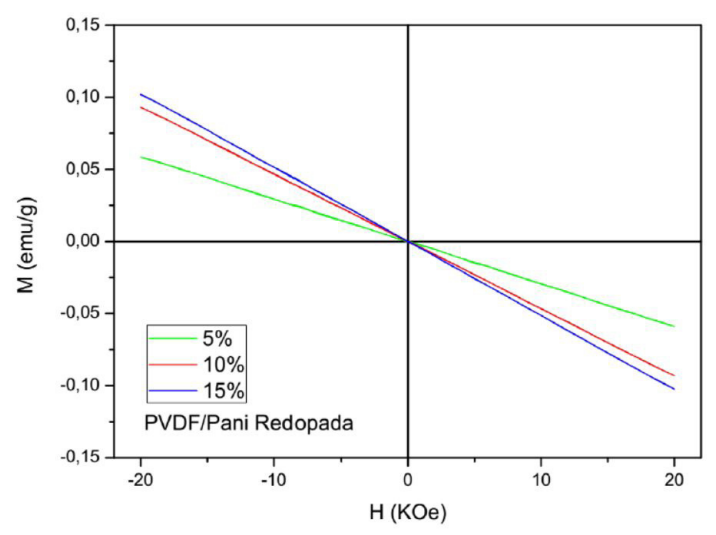

Figura 2. Curvas de magnetização M-H dos filmes dos compósitos de PVDF/Pani/Ni redopado, com 5, 10 e 15\% de partículas de níquel.

Incorporou-se as quantidades de 5, 10, 15, 20, 15 e $30 \%$ (\%) em massa de partículas de níquel em relação a massa da matriz PVDF utilizada na síntese.

Os filmes dos compósitos foram obtidos por prensagem a quente, colocando o material resultante da mistura física entre duas folhas de Kapton sob uma pressão de $30 \mathrm{MPa}$ e a 
uma temperatura de $180^{\circ} \mathrm{C}$. Destas foram obtidas amostras de aproximadamente $180 \mu \mathrm{m}$ de espessura (Figura 3).

Os difratogramas de raios $\mathrm{X}$ foram obtidos em um difratômetro da marca SHIMADZU modelo XRD-6000 com comprimento de onda característico do cobre $\mathrm{K} \alpha-\mathrm{Cu}$ igual a $1,54 \AA$. A análise foi obtida para filmes com um ângulo de incidência de $2 \theta$ de $5^{\circ}$ a $100^{\circ}$.

As micrografias das amostras foram obtidas em um microscópio eletrônico de varredura computadorizado da marca ZEISS modelo EVO LS15, com feixe de elétron de 10 e $20 \mathrm{kV}$.

A condutividade através do método de duas pontas foi medida aplicando uma tensão (V) com uma fonte de tensão e corrente programável digital da Keithley modelo 236 medindo se a corrente (I). A condutividade $(\sigma)$ foi obtida utilizando a Equação 1.

$$
\sigma=\frac{\ell}{A} \cdot \frac{I}{V}
$$

Sendo $\ell$ a espessura da amostra e A, a área da superfície metalizada em ambas as faces, com valor de aproximadamente $\mathrm{A}=5,0 \times 10^{-5} \mathrm{~m}^{2}$.
As análises térmicas foram realizadas em um MDSC 2920 para o DSC e um SDT Q600 para o TGA ambos da TA Instruments, com razão de aquecimento de $10^{\circ} \mathrm{C} / \mathrm{min}$ em atmosfera dinâmica de nitrogênio.

Os ensaios de tração foram realizados em um equipamento Instron-3369 de acordo com a norma ASTM D882 com célula de carga de $500 \mathrm{~N}$.

As caracterizações de magnetização em função do campo magnético externo (M-H) dos filmes PVDF/Pani puros e com a incorporação de 5 e $25 \%$ de partículas de níquel foram realizadas em um magnetômetro de amostra vibrante VSM-ADE EV9-Microsense com campo magnético aplicado na faixa de $\pm 20 \mathrm{kOe}$ à temperatura ambiente.

\section{Resultados}

A partir dos resultados obtidos através da difratrometia de raios $\mathrm{X}$, observa-se que tanto para os compósitos desdopados (Figura 4a) quanto para os redopados (Figura 4b) a presença dos picos característicos do PVDF em $2 \theta$ igual

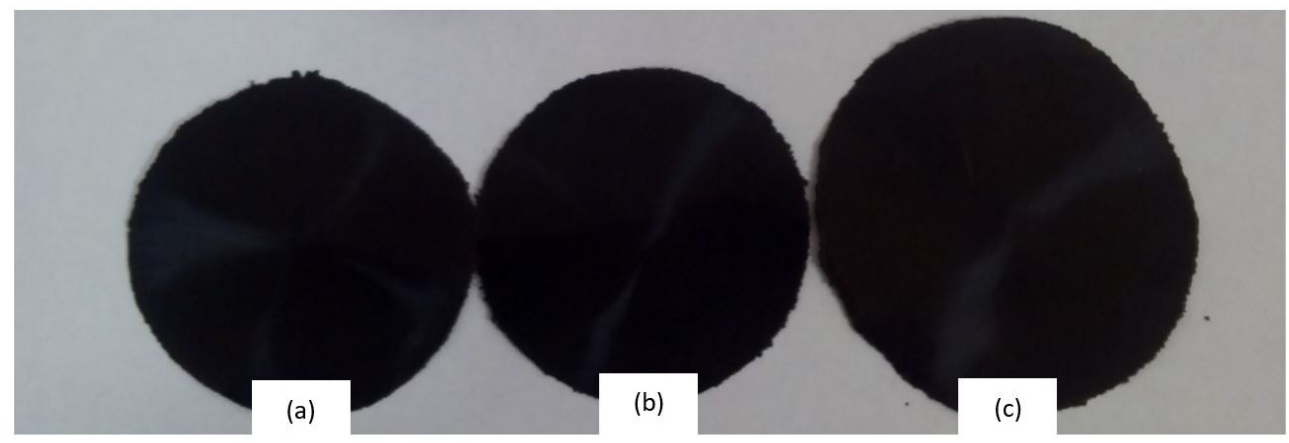

Figura 3. Filmes obtidos após prensagem a quente do compósito PVDF/Pani/Ni desdopado e com (a) 5\%, (b) $15 \%$ e (c) $0 \%$ de partículas incorporadas.

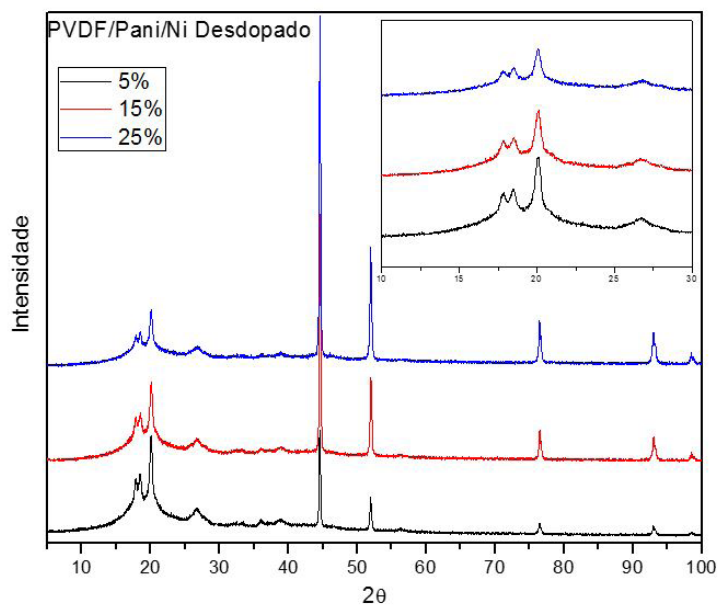

(a)

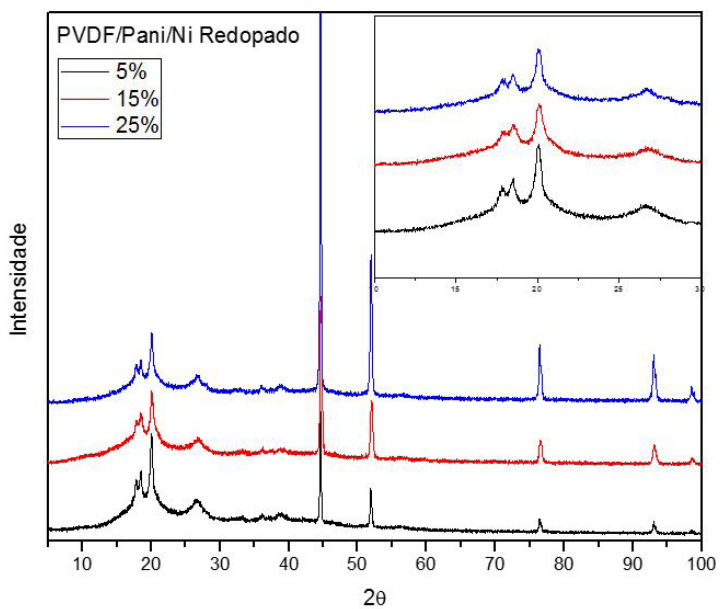

(b)

Figura 4. Difratrometria de raios X do compósito de PVDF/Pani em desdopado em (a) e redopado com $\mathrm{HCl}$ 0,05 em (b) ambos com 5, $15 \%$ e $25 \%$ de partículas de níquel. 
$17.3^{\circ}, 18.3^{\circ}, 19.9^{\circ}, 26.5^{\circ}$ nos quais são característicos da fase $\alpha$ do PVDF (em destaque ao canto).

A presença da Pani tanto desdopada com redopada, assim como as partículas de níquel, independentemente das quantidades de partículas não alterou o padrão da matriz polimérica, verifica-se picos característicos do material [ICSD 4-850] em $2 \theta$ igual a $44.5^{\circ}, 52.89^{\circ}, 76.43^{\circ}, 92.95^{\circ} \mathrm{e}$ $98.46^{\circ}$, estes mais intensos devido ao aumento de conteúdo.

Os termogramas das amostras PVDF/Pani desdopada e redopada com $0,5,15$ e $25 \%$ de partículas de níquel respectivamente são apresentados na Figura 5. Em destaque a termogravimetria para PVDF puro e Pani pura desdopada e redopada, respectivamente.

Para PVDF puro, verifica-se uma única perda de massa em torno de $450^{\circ} \mathrm{C}$ atribuído à degradação térmica do de sua cadeia ${ }^{[32]}$. Com relação à Pani desdopada observa-se dois estágios de perda de massa. O primeiro na faixa de $50-135^{\circ} \mathrm{C}$, com perda de massa de aproximadamente $5 \%$, atribuída a volatilização da água, absorvida pela cadeia polimérica. Um segundo estágio na faixa $400-600^{\circ} \mathrm{C} \mathrm{com}$ perda de massa de $40 \%$ é atribuído à decomposição térmica da cadeia principal do polímero ${ }^{[33]}$.

Para a Pani redopada, verifica-se que a perda de massa ocorre de uma forma praticamente contínua na faixa de $50-600{ }^{\circ} \mathrm{C}$. Na faixa de $50-125^{\circ} \mathrm{C}$ uma perda de massa de
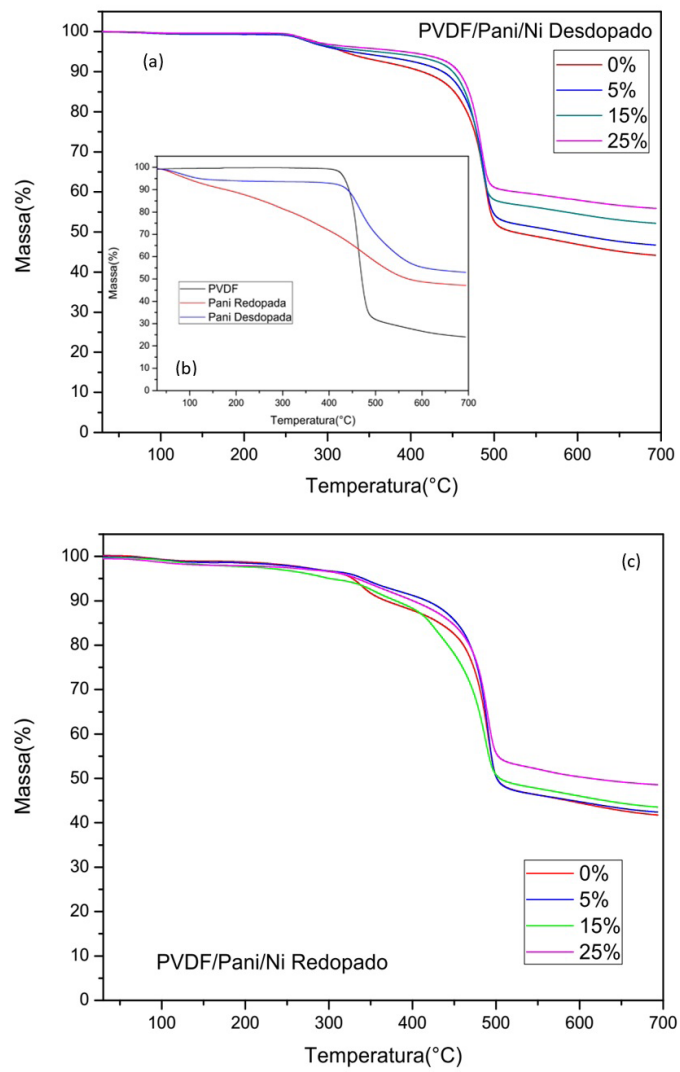

Figura 5. Curvas termogravimétricas de PVDF/Pani desdopada com partículas de níquel em (a) Puras em (b) e da PVDF/Pani redopadaem com partículas de níquel em (c).
$8 \%$ aproximadamente, podemos atribuir a volatilização da água absorvida pelo polímero na forma de umidade. $\mathrm{Na}$ faixa de $135-400{ }^{\circ} \mathrm{C}$ com perda de massa de $20 \%$ pode ser atribuída a volatilização do solvente. Para temperatura de $400^{\circ} \mathrm{C}$ observa-se outro estágio com perda de massa de $25 \%$ que se pode atribuir também à decomposição térmica da cadeia principal da Pani ${ }^{[3,343}$.

Observa-se a presença destes três estágios no compósito, o primeiro na faixa de temperatura de $50{ }^{\circ} \mathrm{C}$ a $125^{\circ} \mathrm{C}$, o segundo de $250{ }^{\circ} \mathrm{C}$ a $400{ }^{\circ} \mathrm{C}$ e o terceiro de $400{ }^{\circ} \mathrm{C}$ a $550{ }^{\circ} \mathrm{C}$ atribuídos a volatilização da água absorvida pelo material (aproximadamente 1\%), a volatilização do solvente e a decomposição térmica da cadeia principal da Pani respectivamente ${ }^{[34]}$.

Isso sugere que pelo fato da amostra redopada apresentar maior quantidade de dopante em sua estrutura, sua perda será maior ${ }^{[33,34]}$ até o início da decomposição térmica da cadeia principal da mistura.

Perdas relativamente menores para o compósito redopado, pois não há as contribuições de resíduo termorrígido, uma vez que a formação de ligações cruzadas é inibida pelo ácido ( $\mathrm{HCl})$ como será observado nas análises de DSC. Nota -se o aumento da formação de resíduo associado ao aumento do conteúdo de partículas de incorporados na matriz.

Os termogramas dos compósitos desdopados e redopado com $0,5,15$, e $25 \%$ de partículas de níquel são apresentados na Figura 6. Verifica a presença de um pico endotérmico em $180{ }^{\circ} \mathrm{C}$, referente à fusão do $\mathrm{PVDF}$ em $180^{\circ} \mathrm{C}^{[35,36]} \mathrm{e}$ um pico exotérmico em $250{ }^{\circ} \mathrm{C}$ referente à formação de ligações cruzadas da Pani desdopada ${ }^{[36]}$.

Para o compósito redopado, nota-se a o mesmo pico endotérmico da fusão da matriz, evidenciando que a presença da Pani como a das partículas não afetam a região cristalina do PVDF, apenas uma diminuição deste, novamente devido ao aumento de conteúdo das partículas, porém sem deslocá-lo.

Nota-se também a ausência da transição exotérmica em $250^{\circ} \mathrm{C}$ como observado para a Pani desdopada, sugerindo que para o compósito redopado a presença do ácido inibe a formação dessas ligações na mesma como citado anteriormente $^{[36]}$.

As micrografias com um aumento de 500x dos compósitos de PVDF/Pani/Ni desdopado e redopado com a incorporação das partículas são apresentados na Figura 7. Observa-se que o material com 5\% de partículas apresenta apenas alguns aglomerados, dispersos aleatoriamente.

Com o aumento da quantidade de partículas entre $15 \%$ e $25 \%$ nota se uma distribuição mais homogênea das partículas no que pode dar ideia da formação de uma rede condutora exclusivamente das partículas.

A Figura 8 apresenta as micrografias com o aumento de 2000x da superfície dos compósito PVDF/Pani/Ni) amostra com $5 \%$ de partículas em (a) e $25 \%$ e em (b) ambos redopados. Verifica-se (em destaque) a alguns defeitos ao longo do filme e com o aumento de conteúdo a formação de aglomerados de partículas (pontos claros).

A condutividade elétrica foi estudada em função da incorporação de partículas de níquel em relação à massa do PVDF puro e na mistura PVDF/Pani desdopada e redopada. 

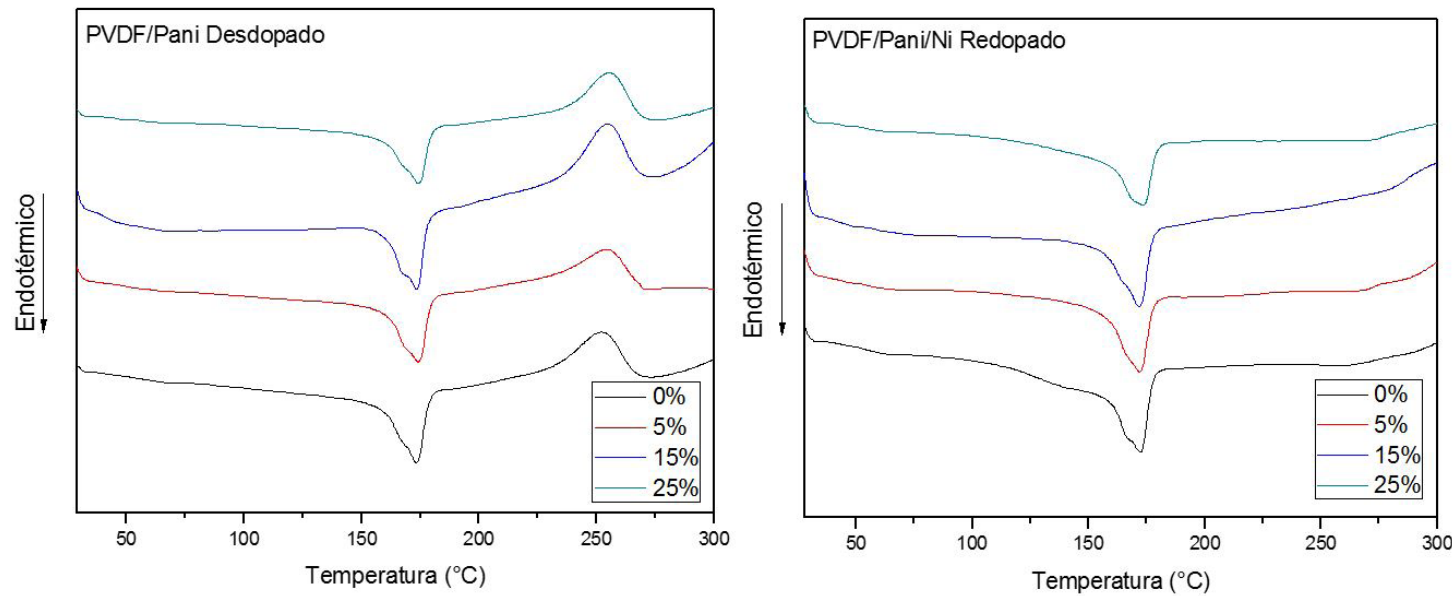

Figura 6. Termogramas do compósito PVDF/Pani/Ni desdopado e redopado com incorporação de $0,5,15$ e $25 \%$ de partículas de níquel.
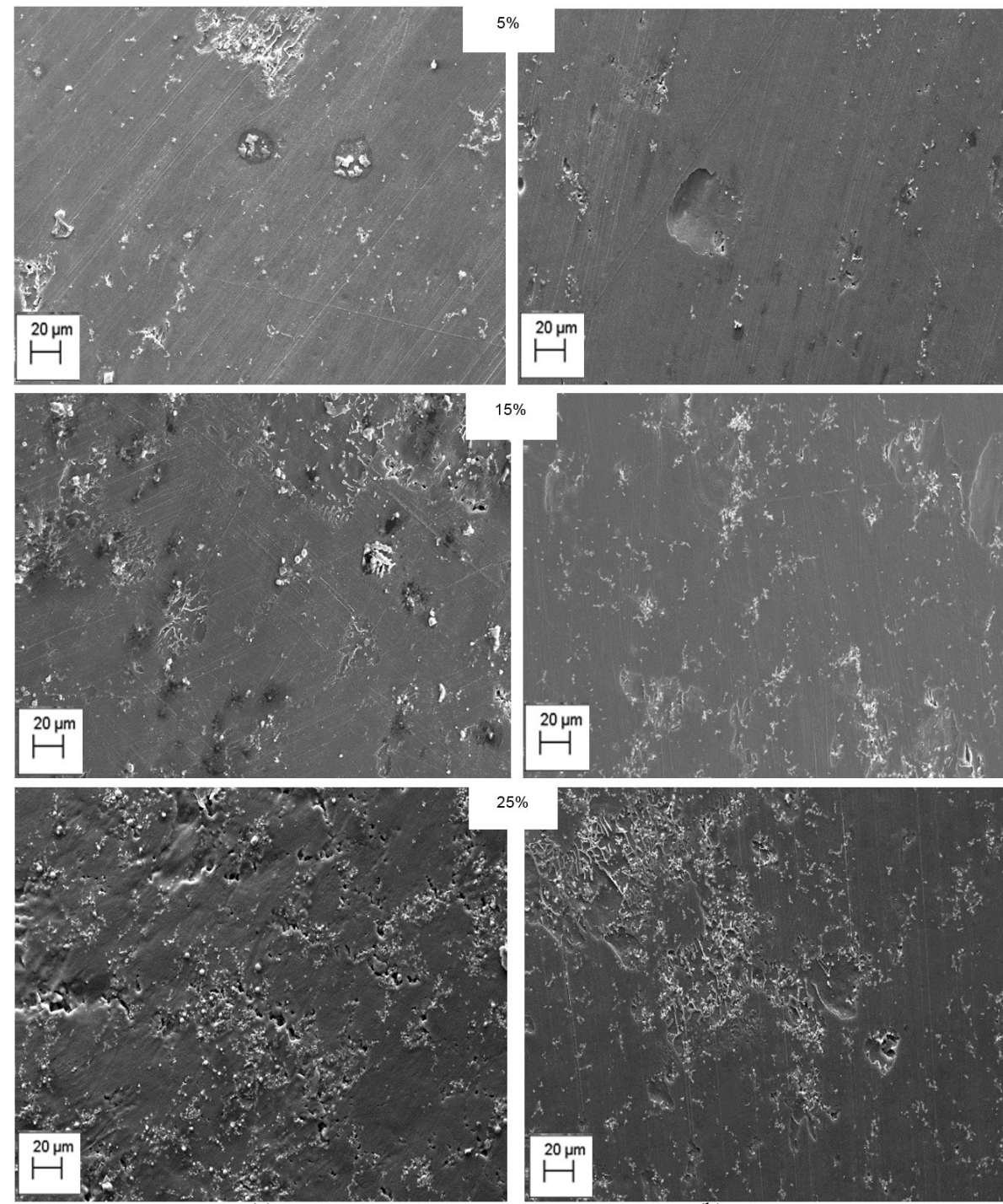

(a)

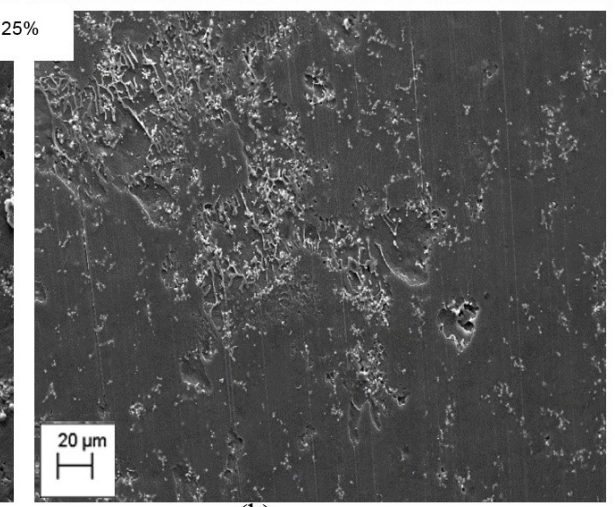

(b)

Figura 7. Micrografias da superfície dos compósitos de PVDF/Pani/Ni com a incorporação de 5\%, 15\% e 25\% de níquel: (a) desdopados e (b) redopados com $\mathrm{HCl}$ 0,05 M, com um aumento de 500x. 

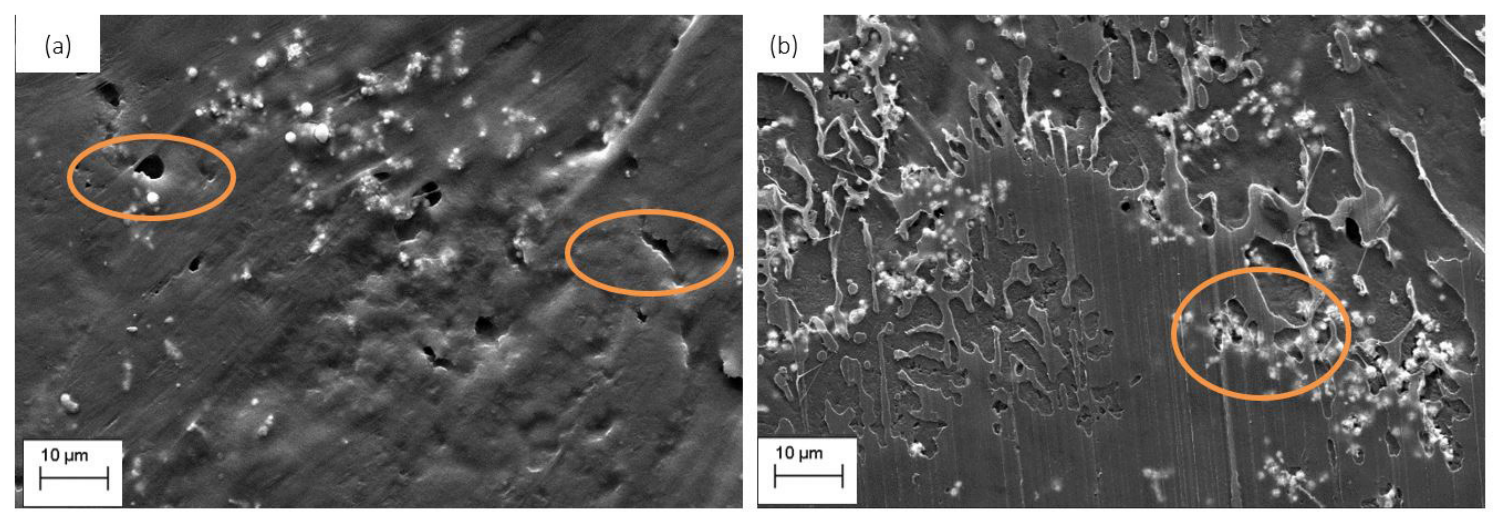

Figura 8. Micrografias da superfície dos compósitos de PVDF/Pani/Ni com a incorporação de (a) $5 \%$ e (b) $15 \%$ de níquel ambos redopados com $\mathrm{HCl}$ 0,05 M, com um aumento de 2000x.

Para o PVDF puro, com uma concentração de níquel abaixo de $15 \%$ o valor da condutividade aumenta lentamente. Acima dessa concentração, entre 15 e 20\%, a condutividade apresenta um limiar de percolação com um salto no seu valor em 7 ordens de grandeza atingindo um valor exclusivamente das partículas de níquel $\left(10^{-4} \mathrm{~S} / \mathrm{cm}\right)$.

Este salto na condutividade é ocasionado pela percolação geométrica, ou seja, pelo contato entre as partículas de níquel $^{[37]}$. A Tabela 1 apresenta os valores obtidos para o matriz PVDF pura com incorporação de partículas.

Ao comparar se os resultados obtidos para incorporação na mistura, verifica-se um aumento gradativo de 3 ordens de grandeza no valor da condutividade entre 5 e $15 \%$ de partículas. Isso sugere que de alguma forma a Pani mesmo que desdopada (isolante), $\left(10^{-10} \mathrm{~S} / \mathrm{cm}\right)$ contribui para o aumento da condutividade do material, havendo dois caminhos condutores distintos no mesmo: (1) um pela percolação elétrica que ocorre com o mecanismo de transporte via tunelamento e saltos mesmo que pequeno, pelos caminhos condutores da Pani e (2) pelo contato entre as partículas de níquel, uma a percolação geométrica, como citado anteriormente ${ }^{[37]}$.

Para o compósito redopado o aumento da quantidade de partículas provoca um decréscimo de uma ordem de grandeza, até valor próximo das partículas de níquel puro $\left(10^{-4} \mathrm{~S} / \mathrm{cm}\right)$.

Este resultado evidencia que, a incorporação das mesmas atrapalham os caminhos condutor da Pani quando esta encontra se no estado condutor, dificultando o processo de condução dos portadores de cargas pelo polímero.

Já com o aumento de partículas passaríamos a ter o aumento do segundo caminho condutor no interior do compósito, como sugerido pelas micrografias (Figura 8b).

Pode-se ainda interpretar devido a variação nos valores de condutividade, que a incorporação de partículas atua como impurezas em relação à Pani ${ }^{[19]}$.

Assim quanto maior a quantidade de partículas como verificado nas micrografias, dá se a ideia que maior será a distância entre as regiões condutoras da Pani e por sua vez admitindo uma condutividade exclusivamente das partículas do níquel, devido à maior formação de aglomerados.
Tabela 1. Condutividade elétrica da matriz PVDF em função da incorporação de partículas.

\begin{tabular}{cc}
\hline \multicolumn{2}{c}{ Condutividade Elétrica (S/cm) } \\
\hline Niquel (\%) & PVDF \\
\hline 0 & $3,219 \times 10^{-12}$ \\
5 & $4,020 \times 10^{-12}$ \\
10 & $8,629 \times 10^{-12}$ \\
15 & $1,545 \times 10^{-11}$ \\
20 & $2,799 \times 10^{-4}$ \\
25 & $8,100 \times 10^{-4}$ \\
30 & $8,247 \times 10^{-4}$ \\
\hline
\end{tabular}

Tabela 2. Condutividade elétrica dos compósitos em função da incorporação de partículas.

\begin{tabular}{ccc}
\hline \multicolumn{3}{c}{ Condutividade Elétrica (S/cm) } \\
\hline \multirow{2}{*}{ Niquel (\%) } & $\begin{array}{c}\text { PVDF/Pani } \\
\text { Desdopada }\end{array}$ & $\begin{array}{c}\text { PVDF/Pani } \\
\text { Redopada }\end{array}$ \\
\hline 0 & $6,842 \times 10^{-12}$ & $1,934 \times 10^{-3}$ \\
5 & $6,727 \times 10^{-11}$ & $1,519 \times 10^{-3}$ \\
10 & $1,610 \times 10^{-10}$ & $1,118 \times 10^{-3}$ \\
15 & $4,971 \times 10^{-9}$ & $8,695 \times 10^{-4}$ \\
20 & $2,357 \times 10^{-4}$ & $5,492 \times 10^{-4}$ \\
25 & $4,362 \times 10^{-4}$ & $5,348 \times 10^{-4}$ \\
30 & $4,762 \times 10^{-4}$ & $5,744 \times 10^{-4}$ \\
\hline
\end{tabular}

A Tabela 2 resume os valores de condutividade elétrica obtidos para o compósito.

Quanto as propriedades mecânicas do compósito, verifica-se que presença da Pani no interior da matriz tanto desdopada quanto redopada promove uma diminuição nos valores de deformação e tensão de ruptura, porém com um aumento no módulo de elasticidade para amostra desdopada, quando comparado com o PVDF puro (Figura 9a).

Este resultado sugere que a Pani desdopada está agindo como um reforço para matriz, limitando de certa maneira o movimento das cadeias do PVDF.

O processo de redopagem, no qual obtemos a mistura com maior condutividade, empobrece o material quanto às suas propriedades mecânicas apresentando menores valores de deformação, aproximadamente $80 \%$, tensão na ruptura, além da diminuição no módulo de elasticidade, quando 

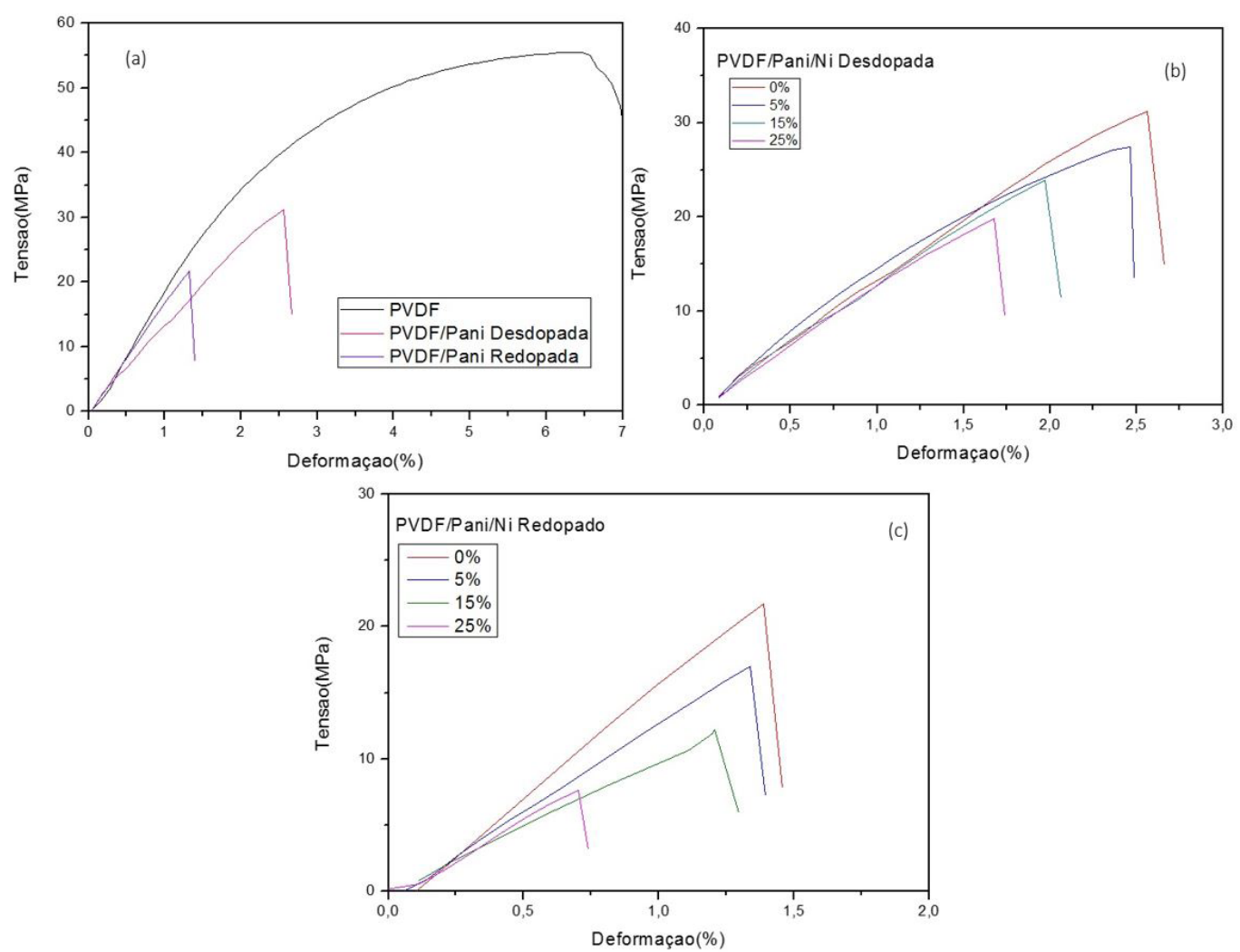

Figura 9. Ensaios da tração dos filmes de: (a) PVDF puro e PVDF/Pani desdopado e redopado; (b) PVDF/Pani/Ni desdopados e com diferentes concentrações de niquel; (c) PVDF/Pani/Ni redopados e com diferentes concentrações de niquel.

comparado aos valores obtidos para a amostra desdopada e o PVDF puro.

Considerando que a Pani pura dopada apresenta baixa resistência mecânica, dificuldade cujo impossibilita teste individuais, pode-se concluir que a presença da mesma no interior da matriz desempenha papel fundamental quanto à interação de suas moléculas com as moléculas da matriz tornando os filmes mais frágil (Figura 9a).

Verifica-se que a incorporação de partículas, assim como o material condutor reduz as propriedades mecânicas do material à medida que a quantidade da mesma aumenta, com a diminuição dos valores da tensão na ruptura, deformação e módulo de elasticidade (Figura 9b). Comportamento semelhante para ambos os estados.

Os valores obtidos da tensão à ruptura, deformação e modo de elasticidade, foram menores para amostras redopadas (Figura 9c). Este resultado eram esperado uma vez que o polímero condutor no estado dopado apresenta propriedades mecânicas reduzidas e quando presente no compósito certamente irá reduzir as propriedades do mesmo, além das micrografias para a amostra com 15\% (Figura 8) já apresentarem falhas ou defeitos ao longo do filmes e nas proximidades dos aglomerados.

A Tabela 3 resume os resultados dos ensaios de tração para o PVDF puro e para os compósitos PVDF/Pani/Ni desdopados, redopados com incorporação 5,15 e $25 \%$ de partículas.
As medidas magnéticas são apresentadas nas Figuras 10 e 11 . Nota-se que antes da incorporação o compósito apresenta um comportamento diamagnético (Figura 10a), na qual a magnetização cai linearmente com o campo magnético externo, orientando seus momentos dipolos, contrário a aplicação deste ${ }^{[38]}$ tal comportamento é fortemente influenciado tanto pela presença da matriz PVDF quanto pela Pani, em ambos os estados.

A partir da incorporação das partículas, o compósito passa a apresentar um comportamento ferromagnético ${ }^{[18-23]}$ (Figura 10), no qual é evidenciado pelo comportamento histerético apresentado (Figura 10c). As magnetizações dos domínios se alinham em função do aumento do campo até um valor de saturação da amostra $\left(\mathrm{M}_{\mathrm{s}}\right)$.

Para o compósito com $5 \%$ de incorporação foram obtidos valores de magnetização de saturação, $M_{\mathrm{s}}=3,16 \mathrm{emu} / \mathrm{g}$. Ao diminuir se o campo aplicado, verifica-se que a curva passa a tocar o eixo das ordenadas, onde associa-se a maioria dos domínios estarem ainda alinhados. Aqui temos a magnetização remanente do material $(\mathrm{Mr}), \mathrm{M}_{\mathrm{r}}=0,49 \mathrm{emu} / \mathrm{g}$ e o campo coercivo $\left(\mathrm{H}_{\mathrm{c}}\right)$, (ponto onde a megnetização é nula) $\mathrm{H}_{\mathrm{c}}=0,084$ KOe. Para o compósito com $25 \%$ de partículas os resultados obtidos foram de $\mathrm{M}_{\mathrm{s}}=9,67 \mathrm{emu} / \mathrm{g}, \mathrm{M}_{\mathrm{r}}=1,53 \mathrm{emu} / \mathrm{g}$ e $\mathrm{H}_{\mathrm{c}}=0,079 \mathrm{KOe}$, respectivamente.

Para o compósito redopado (Figura 11a), a incorporação de $5 \%$ de partículas a amostra apresenta valores de magnetização de saturação $\mathrm{M}_{\mathrm{s}}=1,45 \mathrm{emu} / \mathrm{g}$; e magnetização remanente de $\mathrm{M}_{\mathrm{r}}=0,18 \mathrm{emu} / \mathrm{g}$, além de um campo coercivo 

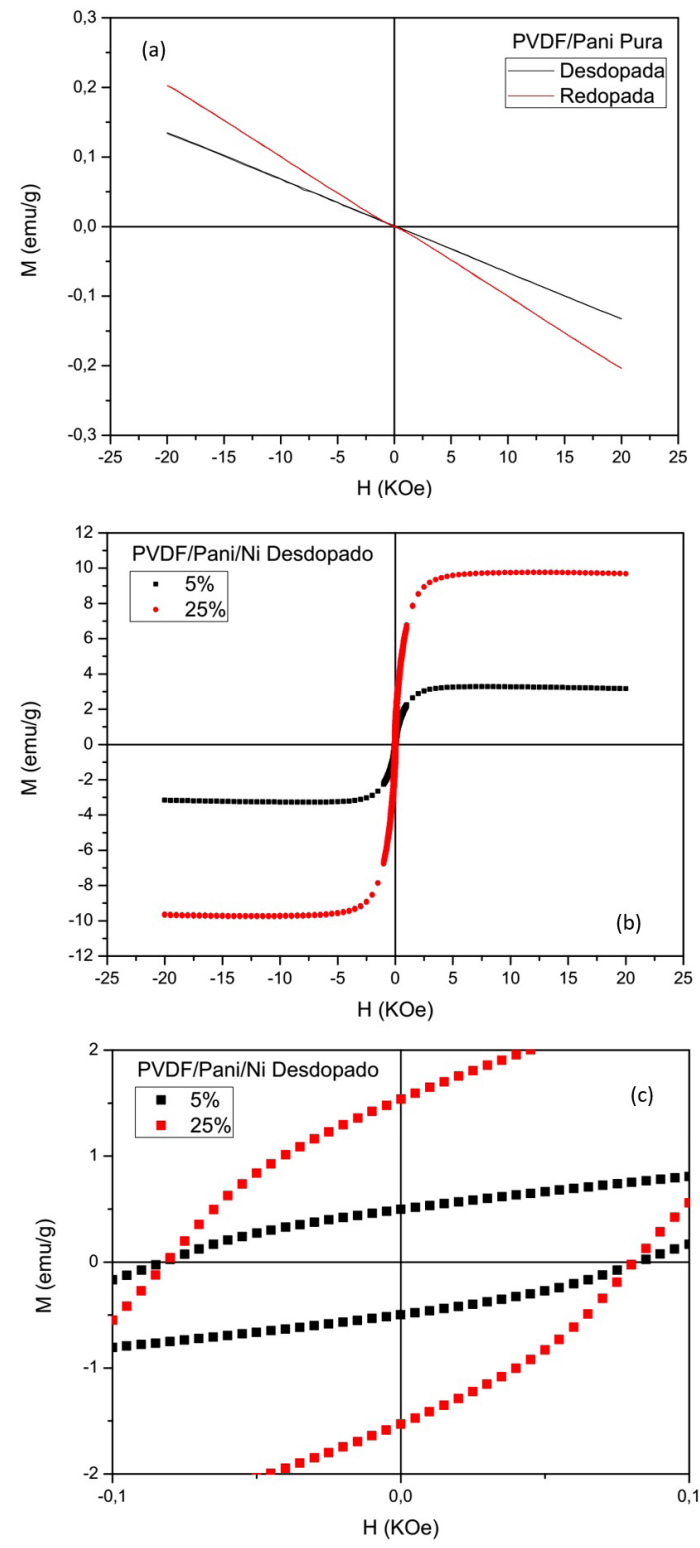

Figura 10. Medidas magnéticas dos filmes de: (a) PVDF/Pani desdopada e redopadado; (b) PVDF/Pani/Ni desdopado e com $5 \%$ e $25 \%$ de partículas de níquel; (c) loop de histereses dos filmes PVDF/Pani/Ni com 5\% e 25\% de partículas.

de $\mathrm{H}_{\mathrm{c}}=0,085$ KOe. Para incorporação de $25 \%$ o compósito passa apresentar maiores valores quanto as magnetizações e campo coercivo; $M_{\mathrm{s}}=8,40 \mathrm{emu} / \mathrm{g} ; \mathrm{M}_{\mathrm{r}}=1,06 \mathrm{emu} / \mathrm{g}$, além dos mesmos vaores de campo coercivo $\mathrm{H}_{\mathrm{c}}=0,084 \mathrm{KOe}$ (Figura 11b), isto muito provavelmente devido a dependência do tamanho da partícula ${ }^{[38]}$.

Observa-se que tanto para o compósito desdopado como redopado, há um aumento nos valores de magnetização em função do aumento do campo magnético externo aplicado, havendo poucas perdas de energia, evidenciados pela o estreitamento na curva de histerese ao longo do eixo central, além de apresentar de baixos campos coercivos ${ }^{[39-44]}$, valores
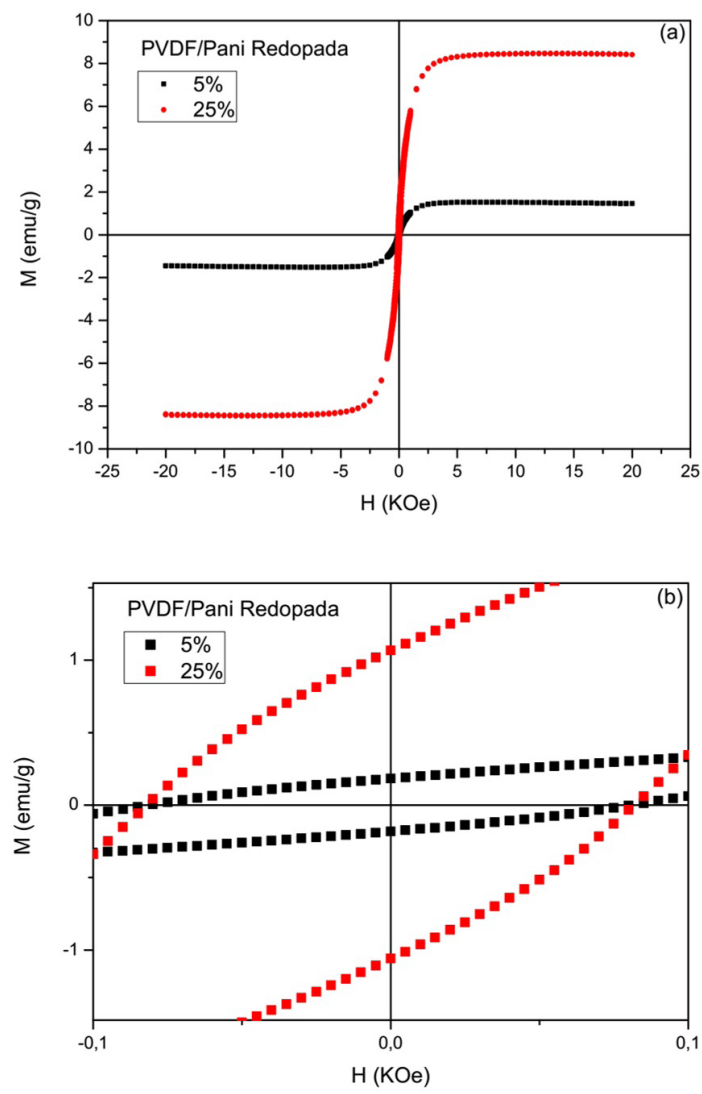

Figura 11. Medidas magnéticas dos filmes de: (a) PVDF/Pani redopadada com $5 \%$ e $25 \%$ de partículas incorporadas; (b) loop de histereses dos filmes PVDF/Pani/Ni com 5\% e 25\% de partículas.

Tabela 3. Resultados dos ensaios de tração para o PVDF puro e para os compósitos PVDF/Pani/Ni desdopados e redopados.

\begin{tabular}{lccc}
\hline \multicolumn{1}{c}{ Amostras } & $\begin{array}{c}\text { (б) Tensão } \\
(\mathbf{M P a})\end{array}$ & $\begin{array}{c}(\boldsymbol{\varepsilon}) \\
\text { Deformação } \\
(\mathbf{\%})\end{array}$ & $\begin{array}{c}\mathbf{E} \\
(\mathbf{G P a})\end{array}$ \\
\hline PVDF & 55,5 & 6,47 & 1,74 \\
PVDF/Pani/Ni desdopado 0\% & 30,9 & 2,56 & 1,96 \\
PVDF/Pani/Ni desdopado 5\% & 27,4 & 2,43 & 1,67 \\
PVDF/Pani/Ni desdopado 15\% & 23,8 & 1,97 & 1,53 \\
PVDF/Pani/Ni desdopado 25\% & 19,8 & 1,67 & 1,34 \\
PVDF/Pani/Ni redopado 0\% & 21,4 & 1,31 & 1,55 \\
PVDF/Pani/Ni redopado 5\% & 17,0 & 1,28 & 1,52 \\
PVDF/Pani/Ni redopado 15\% & 11,7 & 1,18 & 1,47 \\
PVDF/Pani/Ni redopado 25\% & 7,65 & 0,74 & 1,33 \\
\hline
\end{tabular}

$\sigma=$ resistência à tração, $\varepsilon=$ deformação na ruptura e $\mathrm{E}=$ módulo de elasticidade (módulo de Young).

que facilitam a orientação dos domínios magnéticos das partículas e por sua vez da magnetização da amostra.

\section{Conclusões}

Amostras de PVDF/Pani com partículas de níquel foram obtidas através da mistura física das partículas com o pó resultante da sínese química da polimerização da anilina em matriz de PVDF dissolvido em DMF. 
De acordo com os resultados apresentados a obtenção do novo compósito indicam ao mesmo tempo vantagens e desvantagens, quanto a incorporação de maneira extrínseca das mesmas.

As análises obtidos por difratometria de raios X (DRX) mostraram que o PVDF cristalizou-se em sua fase $\alpha$ e que esta foi preservada mesmo com as presenças das partículas de níquel e da Pani, independentemente do estado de desdopado ou redopado.

Análises de DSC mostraram o pico característico de fusão do PVDF nesta fase e que o mesmo não sofreu alteração com as presenças dos demais materiais. Assim como observado pela difratometria não alterou sua configuração e conformação estrutural da matriz, além de uma boa estabilidade térmica verificada através das análises termogravimétricas (TGA).

As micrografias mostraram que para o compósito apresenta uma distribuição mais homogenia com o aumento de conteúdo de partícula ao longo da superfície do material.

A condutividade elétrica do compósito desdopado apresenta um limiar de percolação com um aumento sete ordens de grandeza, alcançando valores de mesma ordem das partículas, enquanto este mesmo aumento de conteúdo, promoveu um decréscimo de uma ordem de grandeza na condutividade do material enquanto este redopado, ou seja, no estado condutor, aumentando as distâncias das regiões condutoras do polímero, atrapalhando os caminhos condutores da Polianilina.

Os resultados de ensaios de tração evidenciam que a presença de partículas atuou de maneira prejudicial as propriedades mecânicas finais do material com a diminuição da resistência a ruptura e sua capacidade em se deformar. Isso ocorreu com maior intensidade para os compósitos redopados devido a Pani estar no estado condutor e apresentar propriedades mecânicas inferiores.

O levantamento magnético mostra que o compósito apresenta um fraco magnetismo, ou um não magnetismo caracterizado por comportamento diamagnético, fortemente influenciado pelos materiais poliméricos. A partir da incorporação das partículas de níquel o material tanto para amostra desdopada como redopada apresentam um comportamento típico de um ferromagnético evidenciado pelos pontos de magnetização e pelos loops de histereses.

A facilitação do comportamento magnético foi alcançada de maneira extrínseca, sem altos níveis de dopagem em meio ácido, quando comparado a literatura, porém com a diminuição da condutividade elétrica e diminuição das propriedades mecânica, evidenciam uma interação não tão vantajosa, resultados que podem acarretar dificuldades na aplicação do mesmo

Sugere-se para o futuro, uma melhor e mais detalhada análise, quanto à aplicabilidade e obtenção do material. Estudos com a mudança nos níveis de dopagem e até mesmo outro tipo de ácido para melhores resultados de condutividade, além da análise do potencial de blindagem eletromagnética, estudo contínuo em polímeros condutores.

\section{Agradecimentos}

Os autores agradecem a CAPES pelo apoio financeiro recebido e aos demais parceiros.

\section{Referências}

1. Mazumdar, S. K. (2002). Composites manufacturing: materials, product, and process. New York: CRC Press LLC.

2. Mamunya, Y. P., Davydenko, V. V., Pissis, P., \& Lebedev, E. V. (2002). Electrical and thermal conductivity of polymers filled with metal powders. European Polymer Journal, 38(9), 1887-1897. http://dx.doi.org/10.1016/S0014-3057(02)00064-2.

3. Strümpler, R., \& Glatz-reichenbach, J. (1999). Conducting polymer composites. Journal of Electroceramics, 3(4), 329346. http://dx.doi.org/10.1023/A:1009909812823.

4. Gustafsson, G., Cao, Y., Treacy, G. M., Klavetter, F., Colaneri, N., \& Heeger, A. J. (1992). Flexible light-emitting diodes made from soluble conducting polymers. Nature, 357(6378), 477-479. http://dx.doi.org/10.1038/357477a0.

5. Fabrizio, M., Mengoli, G., Musiani, M. M., \& Paolucci, F. (1991). Electrochemical characterization of PANI-Nafion membranes and their electrocatalitic activity. Journal of Electroanalytical Chemistry and Interfacial Electrochemistry, 300(1-2), 23-34. http://dx.doi.org/10.1016/0022-0728(91)85381-X.

6. Bousquet, A., Awada, H., Hiorns, R. C., Dagron-Lartigau, C., $\&$ Billon, L. (2014). Conjugated-polymer grafting on inorganic and organic substrates: a new trend in organic electronic materials. Progress in Polymer Science, 39(11), 1847-1877. http://dx.doi.org/10.1016/j.progpolymsci.2014.03.003.

7. Shinar, J., \& Shinar, R. (2011). An overview of organic lightemitting diodes and their applications. In D. L. Andrews, G. D. Scholes \& G. P. Wiederrecht (Eds.), Reference module in materials science and materials engineering - comprehensive nanoscience and technology (pp. 73-107). The Netherlands: Elsevier. http://dx.doi.org/10.1016/B978-0-12-803581-8.092547.

8. Guo, H.C., Ye, E., Li, Z., Han, M.-Y., Loh, X.J. (2016). Recent progress of atomic layer deposition on polymeric materials. Materials Science \& Engineering. C, Materials for Biological Applications, 70(Pt 2), 1182-1191. http://dx.doi.org/10.1016/j. msec.2016.01.093.

9. Oh, M.-C., Chu, W.-S., Shin, J.-S., Kim, J.-W., Kim, K.-J., Seo, J.-K., Lee, H.-K., Noh, Y.-O., \& Lee, H.-J. (2016). Polymeric optical waveguide devices exploiting special properties of polymer materials. Optics Communications, 362(1), 3-12. http://dx.doi.org/10.1016/j.optcom.2015.07.079.

10. Aksimentyeva, O. I., Artym, V. T., Melnik, O. I., \& Plusnina, T. A. (1994). Magnetic properties of conduncting polymers. Acta Physica Polonica, Warsaw, 85(1), 237-240. http://dx.doi. org/10.12693/APhysPolA.85.237.

11. Harun, M. H., Saion, E., Kassim, A., Yahya, N., \& Mahmud, E. H. N. M. (2002). Conjugated conducting polymers: a brief overview. UCSI Academic Journal. Journal for the Advancement of Science \& Arts, 2, 63-68.

12. Gao, C., \& Chen, G. (2016). Conducting polymer/carbon particle thermoelectric composites: Emerging green energy materials. Composites Science and Technology, 124, 52-70. http://dx.doi.org/10.1016/j.compscitech.2016.01.014.

13. Shirakawa, H., Louis, E. J., Macdiarmid, A. G., Chiang, C. K., \& Heeger, A. J. (1977). Synthesis of electrically conducting organic polymers: halogen derivatives of polyacetylene. Journal of the Chemical Society. Chemical Communications, 16(16), 578-580. http://dx.doi.org/10.1039/c39770000578.

14. Bredas, J. L., \& Street, G. B. (1985). Polarons, bipolarons, and solitons in conducting polymers. Accounts of Chemical Research, 18(10), 309-315. http://dx.doi.org/10.1021/ar00118a005.

15. Devine, J. N., Crayston, J. A., \& Walton, J. C. (1999). Synthesis and design of potential polaronic ferromagnets. Synthetic Metals, 101(1-3), 2294-2295. http://dx.doi.org/10.1016/ S0379-6779(98)00635-3. 
16. Onoda, M., Nakayama, H., Morita, S., \& Yoshino, K. (1993). Electrochemicaldoping properties and electronic states of poly(3-phenylthiophene). Journal of Applied Physics, 73(6), 2859-2865. http://dx.doi.org/10.1063/1.353013.

17. Sersen, F., Cik, G., Szabo, L., \& Dlhán, L. (1996). Role of polarons in the antiferromagnetic behavior of poly (3-dodecylthiophene). Synthetic Metals, 80(3), 297-300. http:// dx.doi.org/10.1016/0379-6779(96)80217-7.

18. Barta, P. E., Niziol, S., Le Guennec, P., Pron, A. (1994). Dopinginduced magnetic phase transition in poly(3-Alkylthiophenes). Physical Review B, 50(5), 3016-3024. http://dx.doi.org/10.1103/ PhysRevB.50.3016.

19. Zaidi, N. A., Giblin, S. R., Terry, I., \& Monkman, A. P. (2004). Room temperature magnetic order in an organic magnet derived from polyaniline. Polymer, 45(16), 5683-5689. http://dx.doi. org/10.1016/j.polymer.2004.06.002.

20. Long, Y., Chen, Z., Shen, J., Zhang, Z., Zhang, L., Xiao, H., Wan, M., \& Duvail, J. L. (2006). Magnetic properties of conducting polymer nanostructures. The Journal of Physical Chemistry. B, 110(46), 23228-23233. PMid:17107170. http:// dx.doi.org/10.1021/jp062262e.

21. Pereira, E. C., Correa, A. A., Bulhões, L. O. S., Aleixo, P. C., Nóbrega, J. A., Oliveira, J. A., Ortiz, W. A., \& Walmsley, L. (2011). Polaronic ferromagnetism in conducting polymers. Journal of Magnetism and Magnetic Materials, 226, 20232025. http://dx.doi.org/10.1016/S0304-8853(01)00095-6.

22. Mattoso, L. H. C. (1996). Plástico que conduzem eletricidade: ficção ou realidade? Polímeros: Ciência e Tecnologia, 6, 6-10.

23. Roth, S., \& Graupner, W. (1993). Conductive polymers: evaluation of industrial applications. Synthetic Metals, 57(1), 3623-3631. http://dx.doi.org/10.1016/0379-6779(93)90487-H.

24. Medeiros, E. S., Oliveira, J. E., Consolin-Filho, N., Paterno, L. G., \& Mattoso, L. H. C. (2012). Uso de polímeros condutores em sensores: parte 1: Introdução aos polímeros condutores. Revista Eletrônica de Materiais e Processos,7(2), 62-77.

25. Malmonge, L. F., \& Mattoso, L. H. C. (1995). Electroactive blends of poly (vinylidene fluoride) and polyaniline derivatives. Polymer, 36(2), 245-249. http://dx.doi.org/10.1016/00323861(95)91310-4.

26. Bhadra, S., Khastgir, D., Singha, N. K., \& Lee, J. H. (2009). Progress in preparation, processing and applications of polyaniline. Progress in Polymer Science, 34(8), 783-810. http://dx.doi.org/10.1016/j.progpolymsci.2009.04.003.

27. Lovinger, A. J. (1982). Developments in crystalline polymers (Vol. 1). United Kingdom: Applied Science Publisher Ltda.

28. Nor, N. A. M., Jaafar, J., Ismail, A. F., Mohamed, M. A., Rahman, M. A., Othman, M. H. D., Lau, W. J., \& Yusof, N. (2016). Preparation and performance of PVDF-based nanocomposite membrane consisting of $\mathrm{TiO} 2$ nanofibers for organic pollutant decomposition in wastewater under UV irradiation. Desalination, 391, 89-97. http://dx.doi.org/10.1016/j.desal.2016.01.015.

29. Woo, Y. C., Kim, Y., Shim, W.-G., Tijing, L. D., Yao, M., Nghiem, L. D., Choi, J.-S., Kim, S.-H., \& Shon, H. K. (2016). Graphene/PVDF flat-sheet membrane for the treatment of RO brine from coal seam gas produced water by air gap membrane distillation. Journal of Membrane Science, 513, 74-84. http:// dx.doi.org/10.1016/j.memsci.2016.04.014.

30. Malmonge, L. F., Lopes, G. A., Langiano, S. C., Malmonge, J. A., Cordeiro, J. M. M., \& Mattoso, L. H. C. (2006). A new route to obtain PVDF/PANI conducting blends. European Polymer Journal, 42(11), 3108-3113. http://dx.doi.org/10.1016/j. eurpolymj.2006.08.002.

31. ASM International. (2000). ASM specialty handbook nickel, cobalt, and their alloys (06178G). San Francisco: J. R. Davis \& Associates.
32. Zulfiqar, S., Zulfiqar, M., Rizvi, M., Munir, A., \& McNeill, I. C. (1994). Study of the thermal-degradation of polychlorotrifluoroethylene, poly(vinylidene fluoride) and copolymers of chlorotrifluoroethylene and vinylidene fluoride. Polymer Degradation \& Stability, 43(3), 423-430. http://dx.doi. org/10.1016/0141-3910(94)90015-9.

33. Gomes, E. C., \& Oliveira, M. A. S. (2012). Chemical polymerization of aniline in hydrochloric acid $(\mathrm{HCl})$ and formic acid $(\mathrm{HCOOH})$ media. Differences between the two synthesized polyanilines. American Journal of Political Science, 2(2), 5-13. http://dx.doi.org/10.5923/j.ajps.20120202.02.

34. Palaniappan, S., \& Narayana, B. H. (1994). Conducting polyaniline salts: thermogravimetric and differential thermal analysis. Thermochimica Acta, 237(1), 91-97. http://dx.doi. org/10.1016/0040-6031(94)85187-5.

35. Liu, Z., Maréchal, P., \& Jérôme, R. (1997). DMA and DSC investigations of the $\beta$ transition of poly(vinylidene fluoride). Polymer, 38(19), 4925-4929. http://dx.doi.org/10.1016/S00323861(96)01074-9.

36. Pandey, S. S., Gerard, M., Sharma, A. L., \& Malhotra, B. D. (2000). Thermal analysis of chemically synthesized polyemeraldine base. Journal of Applied Polymer Science, 75(1), 149-155. http://dx.doi.org/10.1002/(SICI)10974628(20000103)75:1<149::AID-APP17>3.0.CO;2-X.

37. Silva, M. J., Kanda, D. H. F., \& Nagashima, H. N. (2012). Mechanism of charge transport in castor oil-based polyurethane/ carbon black composite (PU/CB). Journal of Non-Crystalline Solids, 358(2), 270-275. http://dx.doi.org/10.1016/j. jnoncrysol.2011.09.032.

38. Suwanwatana, W., Yarlagadda, S., \& Gillespie Jr., J. W. Influence of particle size on hysteresis heating behavior of nickel particulate polymer films. Composites Science and Technology, 66, 2825-2836. http://dx.doi.org/10.1016/j. compscitech.2006.02.033.

39. Moskowitz, B. M. (2006). Hitchhiker's guide to magnetism. 3rd ed. Minneapolis: University of Minnesota.

40. Zhang, Y., Qi, S., Zhang, F., Yang, Y., \& Duan, G. (2011). Preparation and magnetic properties of polymer magnetic composites based on acrylate resin filled with nickel plating graphite nanosheets. Applied Surface Science, 258(2), 732-737. http://dx.doi.org/10.1016/j.apsusc.2011.08.001.

41. Ko, J. M., Park, D.-Y., Myung, N. V., Chung, J. S., \& Nobe, K. (2002). Electrodeposited conducting polymer-magnetic metal composite films. Synthetic Metals, 128(1), 47-50. http:// dx.doi.org/10.1016/S0379-6779(01)00566-5.

42. Chen, W., Li, X., Xue, G., Wang, Z., \& Zou, W. (2003). Magnetic and conducting particles: preparation of polypyrrole layer on $\mathrm{Fe}_{3} \mathrm{O}_{4}$ nanospheres. Applied Surface Science, 218(1- 4), 215221. http://dx.doi.org/10.1016/S0169-4332(03)00590-7.

43. Konyushenko, E. N., Kazantseva, N. E., Stejskal, J., Trchova, M., Kovarova, J., Sapurina, I., Tomishko, M. M., Demicheva, O. V., \& Prokes, J. (2008). Ferromagnetic behaviour of polyaniline-coated multi-wall carbono nanotubes containing nickel nanoparticles. Journal of Magnetism and Magnetic Materials, 320(3- 4), 231-240. http://dx.doi.org/10.1016/j. jmmm.2007.05.036.

44. Nandapure, B. L., Kondawar, S. B., \& Nandapure, A. L. (2012). Magnetic properties of nanostructured cobalt and nickel oxide reinforced polyaniline composites. International Journal of Computers and Applications, 2, 9-14.

Enviado: Ago. 14, 2015

Revisado: Jun. 14, 2016

Aceito: Jun. 29, 2016 